\title{
MUCIDS: an operative $C$ environment for acquisition and processing of polarized-light scattered from biological specimens
}

\author{
A.Diaspro, P.Scelza and C.Nicolini
}

\begin{abstract}
In this work, we describe a software package, MUCIDS, completely developed in our laboratory, for acquisition and processing of differential polarization light-scattering data from specimens of biophysical interest. MUCIDS is a C environment that manages the whole activity of an instrument used for measurements of Mueller matrix scattering elements. It allows one to capture, analyse, process and display data from this or from other similar light-scattering experiments. The entire system is suitable for routine measurements in a general biophysical (or microbiological) laboratory because of its easy handling and maintenance. The software was written in Clattice and will run on IBM personal computers and similar. It uses $I B M / D A C$ and $G P I B / I B M$ interface cards.
\end{abstract}

\section{Introduction}

The light scattered by biological specimens contains a wealth of important information which can be extracted by measuring the properties of the light before and after scattering (Bohren and Huffmann, 1983). Within the last 15 years, several workers have been concerned with the scattering of light by specimens of biophysical interest such as chromatin-DNA, microorganisms, cells (Bicket et al., 1976; Nicolini et al., 1976, 1987, 1988; Nicolini and Kendall, 1977; Bicket and Stafford, 1981; Maestre et al., 1982; Salzman and Gregg, 1983; Nicolini, 1986; Garab et al., 1988).

We have concentrated our research on circular intensity differential scattering (CIDS) and a few other scattering parameters (Bustamante et al., 1983; Zietz et al., 1983; Belmont et al., 1985; Diaspro and Nicolini, 1987a,b; Diaspro et al., 1990).

CIDS, which is related to the preferential scattering of right versus left circularly polarized light, has been proven to be a powerful biophysical tool to study the higher-order structure of large biopolymers, in vitro and in situ (Nicolini et al., 1976, 1988; Nicolini and Kendall, 1977; Maestre et al., 1982) and to discriminate micro-organisms in solution (Bicket et al., 1976; Salzman and Gregg, 1983).

In recent years we have designed and built an instrument able to measure CIDS and the other scattering parameters as a

Institute of Biophysics, School of Medicine, University of Genova, Via Giotto 2, 16153 Genova. Sestri Ponente, Italy function of the scattering angle, within the framework of microbiology and molecular biology (Diaspro and Nicolini, 1987b; Nicolini et al., 1988; Diaspro et al., 1990).

Since the complete characterization of scattered light can be efficently described in the context of Stokes vectors and Mueller matrices (Bohren and Huffmann, 1983), we have developed a software environment useful both to control measurements and to extract scattering parameters in terms of Mueller elements.

The elements $S_{14}$, strictly connected with CIDS, and $S_{34}$ are known to be sensitive to minor changes in biological structures at cellular and subcellular levels. Furthermore they yield different angular patterns in different micro-organisms. For this reason we probe them in the study of important parameters related to the structure and dynamics of biopolymers, such as nucleosomes and polynucleosomes, both in vitro and in situ (Diaspro et al., 1990).

The elements $S_{11}$ and $S_{33}$ are also significant regarding knowledge of the amplitude of the light scattered, which is related to the dimension of the sample, and they can be used for normalization purposes.

In particular, $S_{11}$ is the total intensity scattered from the sample, part of the DC component of the signal, and $S_{33}$ is related to the $\mathrm{AC}$ component of the signal, as described elsewhere (Bohren and Huffmann, 1983; Diaspro and Nicolini, 1987b; Diaspro et al., 1990).

For details on the Mueller matrix elements and their biophysical significance we refer the reader to some of the cited references (Bohren and Huffmann, 1983; Bustamante et al., 1983; Nicolini, 1986; Garab et al., 1988; Johnston et al., 1988; Nicolini et al., 1988).

\section{Hardware and software specifications}

The whole system architecture can be summarized in three fundamental blocks: (i) the 'CIDS machine'; (ii) the computer interface; and (iii) the operative software for acquisition, analyses and display of data.

The 'CIDS machine', described in detail elsewhere (Diaspro and Nicolini, 1987b; Nicolini, et al., 1987; Diaspro et al., 1990), has been built at relatively moderate cost but offers reasonable accuracy and good reproducability. 


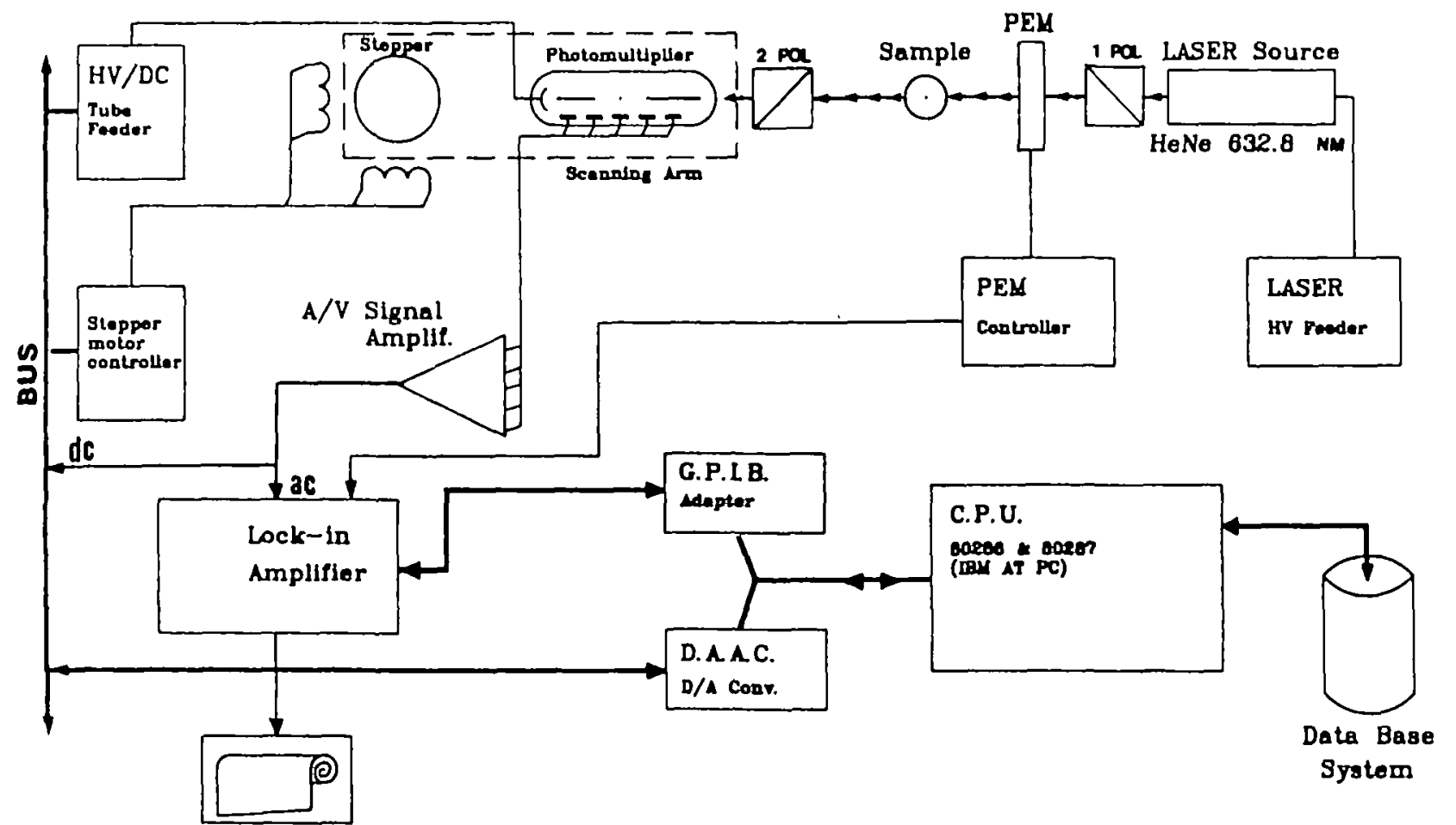

Chart recorder

Fig. 1. Schematic diagram of the hardware architecture managed by MUCIDS. The diagram shows the mult-stage A/V signal amplifier which splits the signal into its DC and AC components to be sent respectively to a signal bus and to the lock-in amplifier; the bus, sketched in the figure, controls the flow from/to the DAC card of the I/O signals, narnely tube voltage regulation and stepper motor pulses as output, and the DC part of the scattering signal as input. MUCIDS, during the acquisition sessions, synchronizes the GPIB and DAC activities.

\section{Hardware}

Hardware has been realized via the 80286 microprocessor (IBM AT), using DAC-IBM and GPIB cards, and is schematically shown in Figure 1.

The light scattered from the sample and transduced by a photomultiplier tube is sent to a transimpedance preamplifier. The preamplifier separates and amplifies the $\mathrm{DC}$ from the $\mathrm{AC}$ components: the former is directly computer connected with an analogue/digital (A/D) multichannel board (IBM-DAC), while the latter is sent to a lock-in-amplifier (5207 EG\&G) to increase the signal-to-noice ratio of the $\mathrm{AC}$ components of interest. In our case the $\mathrm{AC}$ components oscillate at 50 and 100 $\mathrm{kHz}$ and are physically induced by a photoelastic modulation (PEM) of the states of light polarization (Diaspro and Nicolini, 1987b; Diaspro et al., 1990).

The lock-in-amplifier is computer-controlled via a GPIB card. In addition, a driver for a stepper-motor (MAE, Offanengo), completely computer controlled and inter-faced via a $\mathrm{DAC}$ board, has been used. The DAC card operates with a 12 bit resolution $\mathrm{A} / \mathrm{D}$ converter, $100 \mathrm{Mohm}$ minimum input resistance and $200 \mathrm{pF}$ maximum input capacitance. The setting time for each channel, in acquisition, is a maximum of $20 \mathrm{~ms}$ and the throughput to memory is guaranteed for 15000 conversions/s. The lock-in amplifier is usually set to a time constant of 100 $\mathrm{ms}(12 \mathrm{~dB} / \mathrm{oct})$ and receives the signal reference directly from the PEM controller. Demodulation occurs at 50 and $100 \mathrm{kHz}$ and the IEEE-488 protocol is used for input/output computer communications.

The IBM DAC and GPIB cards are directly plugged into the IBM AT and the connections are provided by a control adapter distribution panel for the DAC and by a IEEE-488 dedicated line for the GPIB. Other multifunction I/O cards are suitable (i.e. AT-MIO-16 and MC-MIO-16, National Instruments) to be utilized and managed by this package with a minimum of changes at library level.

The IBM AT is equipped with $0.64 \mathrm{Mb}$ of internal memory, a hard disk with a capacity of $20 \mathrm{Mb}, 1.2 \mathrm{Mb}$ floppy disk driver, a removable tape memory with a capacity of $60 \mathrm{Mb}$ and a 80287 maths coprocessor for arithmetic operations. 


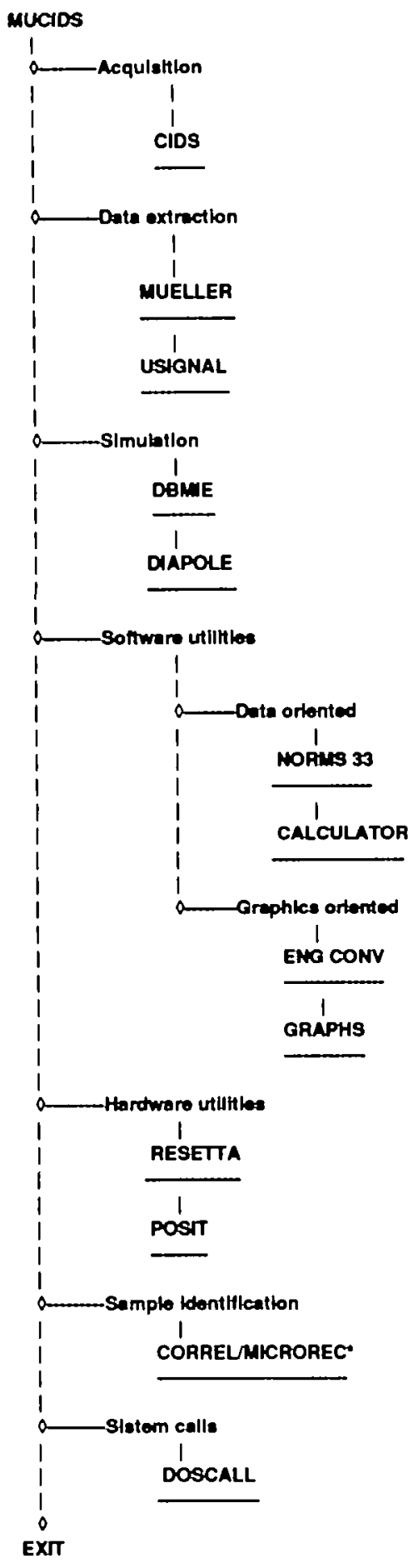

Fig. 2. Summary of the software activities managed by MUCIDS.

\section{Software}

All software, running under the DOS 3.1 operating system, has been written in C-lattice except for a simulation package (in Professional FORTRAN) and some utilities to reset the DAC board and to position the goniometer (BASIC).
All data are properly formatted to be displayed with commercial packages (e.g. Energraphics or similar).

\section{Program description}

\section{Global structure}

MUCIDS is a user interface based on a case control structure that manages the on-line and off-line operations as reported in Figure 2.

The program's capabilities can be summarized as follows:

(a) Acquisition of differential polarization scattering parameters (CIDS).

(b) Extraction of Mueller parameters and file generation for graphic visualization (MUELLER).

(c) Extraction of noise and normalization parameters (USIGNAL).

(d) Conversion of harmonic components, from original data at 50 and $100 \mathrm{KHz}$, for graphic display (ENGCONV).

(e) DAC card reset (RESETTA).

(f) Rotating goniometer absolute repositioning (POSIT).

(g) Data normalization for $\mathrm{S}_{33}$ element (NORMS33).

(h) Simple data operation $(+, *, /,-)$ for rescaling graphics (CALCULATOR).

(i) Simulation of scattering elements for microspheres within the framework of Mie and Rayleigh theories (DBMIE).

(1) Simulation of CIDS data for chromatin-DNA in terms of multiple dipole scattering treated within the framework of classical electrodynamics (DIAPOLE).

(m) Calls for graphic packages: Energraphics, Chart, 3D, etc. (GRAPHS).

(n) DOS utilities, system calls: directories, disk occupation (DOSCALL).

$(o-r)$ Free for future extensions.

(s) Signal analysis for recognition purposes through correlation and autocorrelation techniques, implemented through FFT and IFFT alghorithms (CORREL).

(t) A Turbo Prolog expert system for identification of micro-organisms based on MUCIDS data is in progress (MICROREC).

(z) Exit to DOS (EXIT).

\section{Routine specifications}

The acquisition program, CIDS, is structured as shown in Figure 3. It needs a file data as input to know the scattering angles at which the signal has to be acquired and how to set the electronic instrumentation: LIA, PEM, PMT, amplifiers.

An example of the I/O with the user is reported in Figure 4. The raw data are stored in 'out' files, which have the format shown in Figure 5.

In order to isolate single elements of the Mueller matrix, 


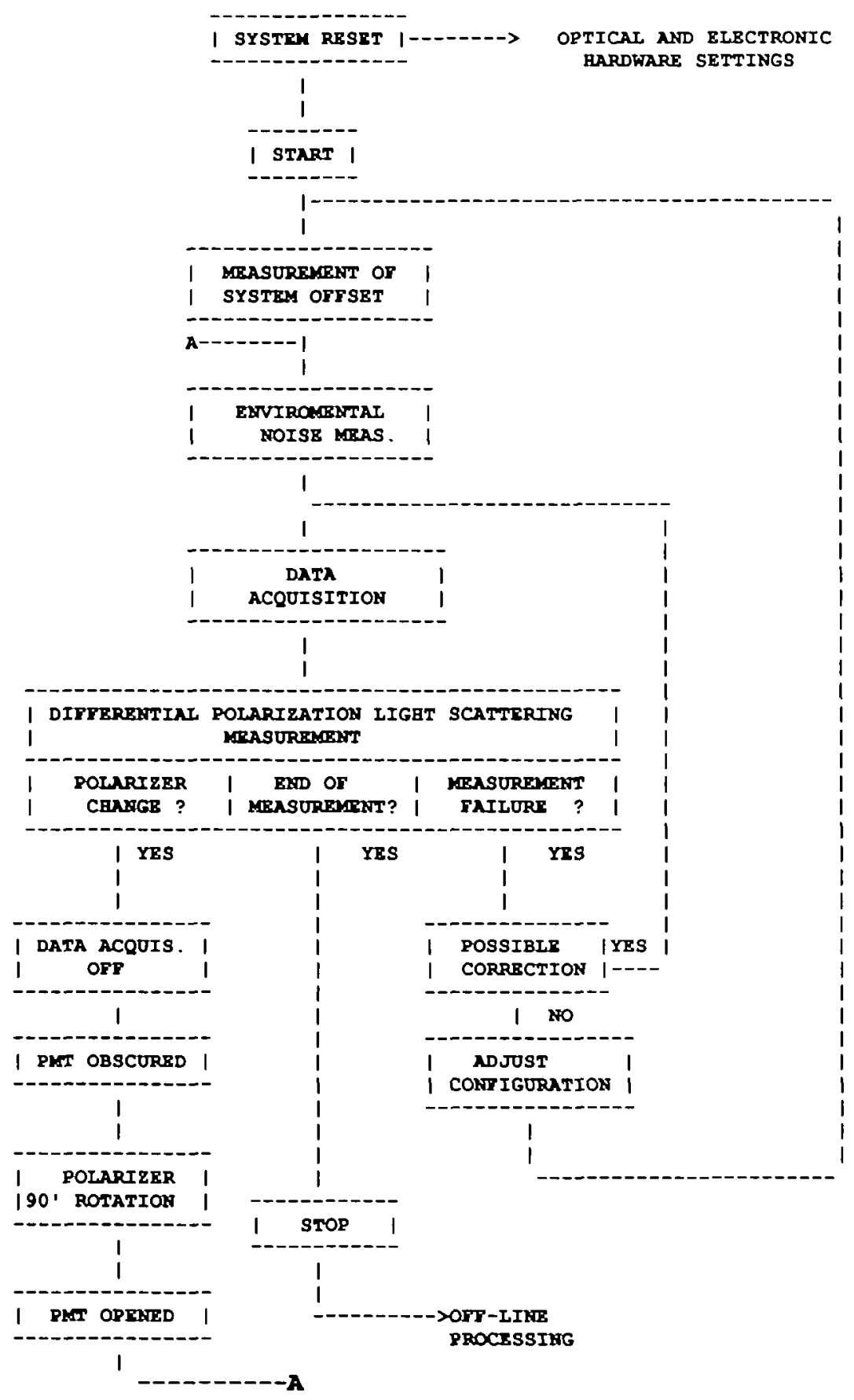

Fig. 3. Schematic flowchan of the on-line activity of the acquisition program.

algebreic operations are performed on the 'out' files, using MUELLER:

(i) Subtraction of the $50 \mathrm{kHz}$ signals, due respectively to $+45^{\circ}$ and $-45^{\circ}$ analyzer orientation, to obtain a value proportional to $S_{14}$.

(ii) Summation as above to extract the $S_{34}$ element.

(iii) The same operations cited above on the $100 \mathrm{kHz}$ signals for parameters proportional (respectively) to $S_{33}$ and $S_{13}$. (iv) Extraction of the $S_{11}$ element using previous data and the DC component of the signal.

(v) Data normalization for $S_{11}$ and for $S_{33}(35)$.

Data can be displayed in terms of harmonic components (Figure 6) or Mueller elements, normalized for $S_{11}$ (Figure 7B) or unnormalized (Figure 7A). 


\section{BIOPTYICS LABOPATOFY GENDA}

CIDG MEATREENT BYgTEM

Qurrent time: Mon Fab 27 04:16:07 1989

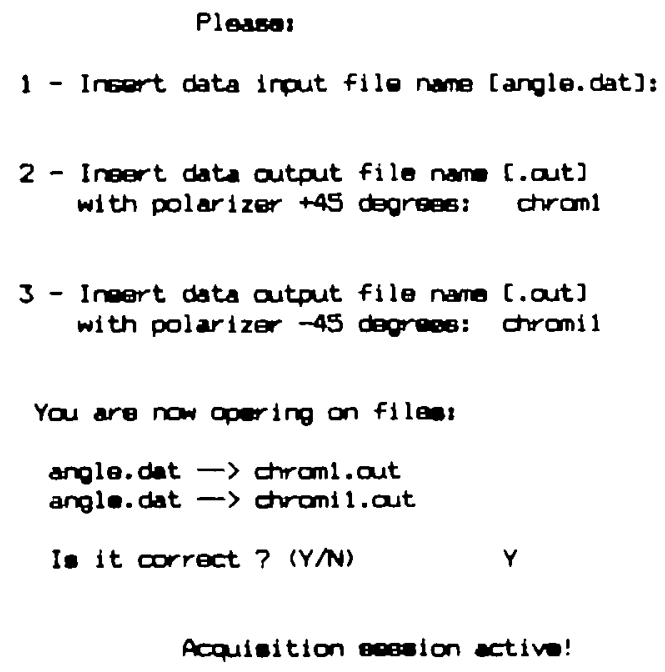

Fig. 4. $1 / O$ user interface for the acquisition activity.

MUELLER can be programmed, through a data file, to take into account different optical situations.

The prediction programs, DBMIE and DIAPOLE, simulate both light scattering from microspheres, within the framework of Rayleigh, Rayleigh-Gans and Mie theories (Bohren and Huffmann, 1983), and differential polarization light scattering from one of the most important biopolymers, chromatin-DNA (Diaspro and Nicolini, 1987).

Mueller elements can be normalized for the element $S_{33}(35)$ by NORMS33 (Figure 7C). CALCULATOR allows the user to scale data or to normalize them by other parameters.

Two packages, partially integrated in MUCIDS, are under development for angular pattern recognition and identification of micro-organisms. The former uses auto- and cross-correlation techniques based on the Fourier transformation of angular sequences from a set of well-known samples constituting the databases. When an unknown sample is acquired it is crosscorrelated with database samples and the recognition is based upon similarities with their autocorrelation patterns.

The latter is an expert system, based on a frame structure, that mainly utilized differential polarization scattering data to identify possible infections.

\section{Discussion}

\section{The goals of the software}

Our purpose was to create a complete software package to simplify the use, acquisition, analysis and display of differential polarization light-scattering data. The software had to integrate different aspects such as theoretical predictions, data acquisition, signal processing, data analysis, display and data comparison. All steps-experimental or theoretical-utilized in the software and all intermediate calculations had to be accessible to the user, in particular at this developmental stage of such a relatively new technique, to prevent the software from being a 'black box'. The display of data had to allow different output levels to obtain a complete description and analysis of the light scattered from the sample.

Furthermore, since MUCIDS is continually developing as the requirements of its users become apparent, the system had to be expandable.

\section{Flexibility}

MUCIDS has been designed and implemented for flexible data acquisition, flexible organization and presentation of data files, and flexibility in how the data can be analysed and correlated with each other.

\section{Ease of use}

All of the MUCIDS routines are accessed through a set of menus, each of which is self-explanatory concerning the selected activity. MUCIDS requires no knowledge of software or of hardware other than the ability to use the keyboard as an interface, to place the sample correctly and to decide the desired experimental configuration.

\section{Portability}

The portability of the major part of the software managed by MUCIDS and of the MUCIDS kernel itself is guaranteed by being written in $\mathrm{C}$, which is a highly portable language. The software can be easily installed on IBM or IBM-compatible personal computers at different levels. The theoretical section or the data reduction and display sections can be installed independently from the acquisition section, which is dependent on two standard cards (DAC, GPIB). The experiments would work utilizing other computer systems, apart from that described here, and the user has only to pay attention to utilize the related libraries correctly. In the worst case a conversion code has to be used in order to utilize MUCIDS source files and different function calls for the acquisition cards (GPIB and multifunction I/O cards).

\section{Expandability}

The use of a program shell, the MUCIDS kernel, facilitates the development of new routines. As described above, future expansion has been planned for in the current version. 


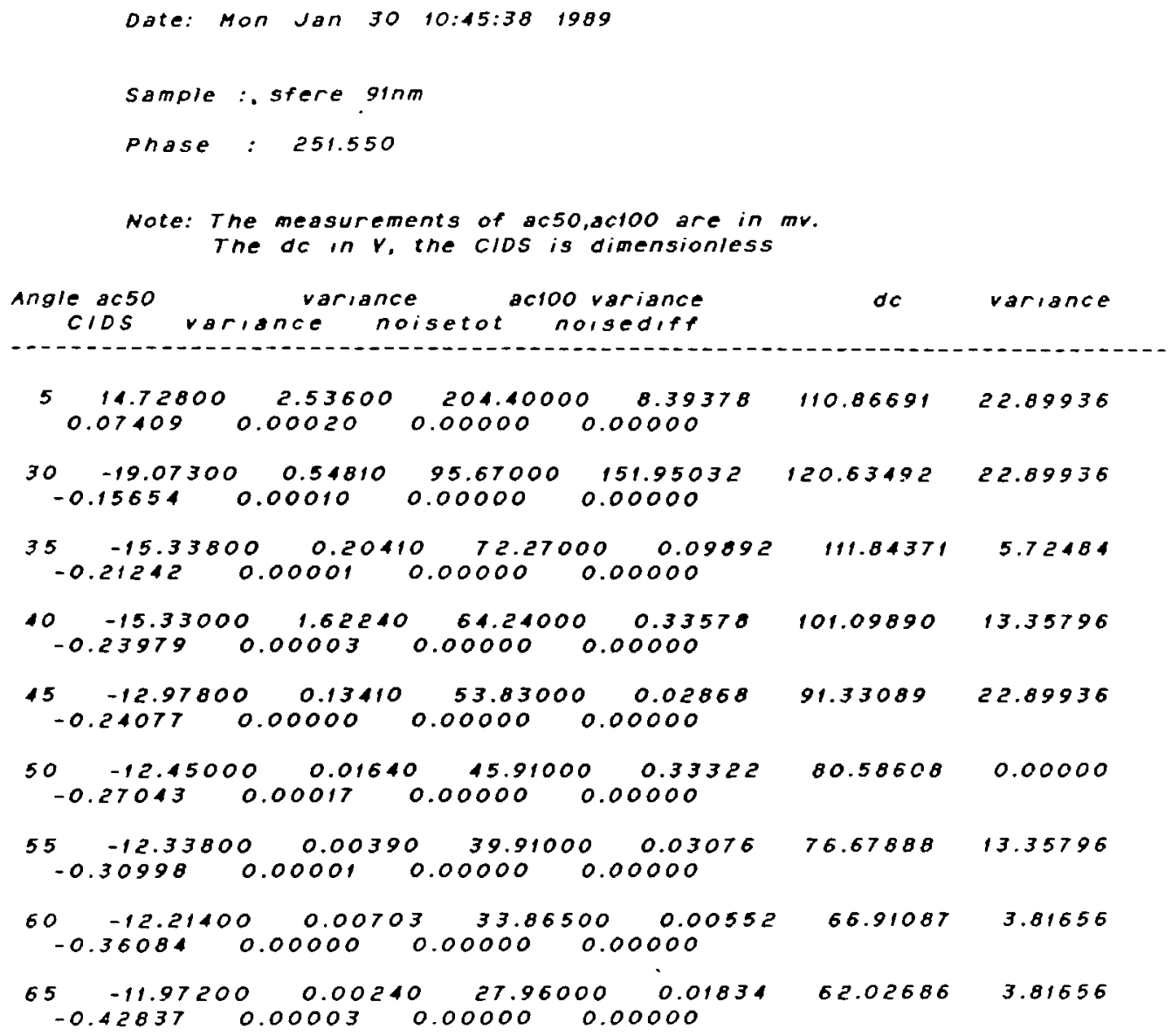

Fzg. 5. Format of the data acquired durng the experimental sessions In this example the noise value reported is zero because the noise test control has been switched off. This can be arbitrarly done in order to reduce the acquisition time needed for the current session.

\section{Conclusion}

We have described here interactive software for acquisition, processing, analysis and display of differential polarization lightscattering data from biological specimens. The programs are transportable and easy to use. MUCIDS allows one to manage both theoretical and experimental activities with high flexibility and makes all the complex hardware and software transparent for the user. The format for the data is simple so that the program will run with data from other acquisition systems. MUCIDS is, in fact, highly modular in the sense that one can easily install only the interesting parts and skip the others, without detrimental consequences on the global architecture of the system.

The capabilities of the software include on-line and off-line operations, namely: data acquisition; data extraction in terms of Mueller parameters, harmonic components, noise; simulation for standard samples, such as microspheres, using classical approaches; prediction of chromatin-DNA behaviour; software

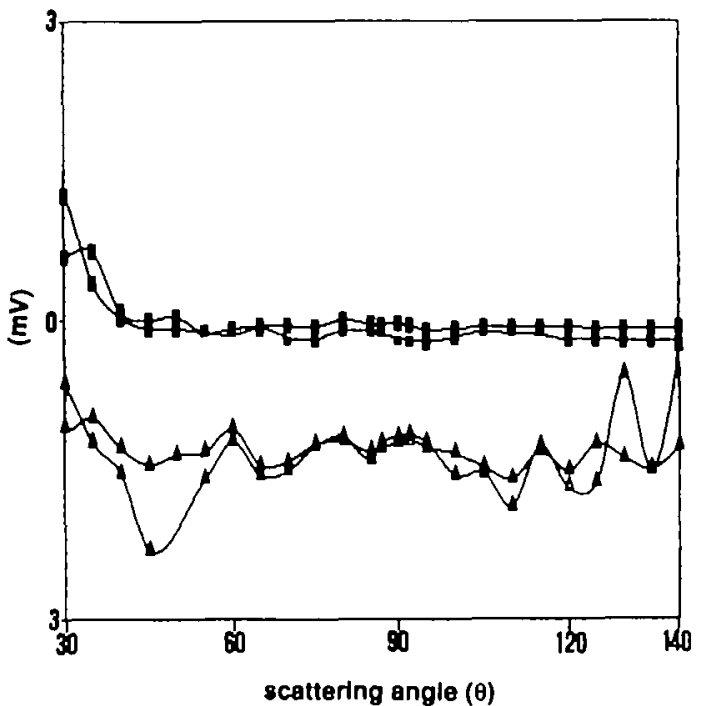

Fig. 6. Display of the data in terms of the harmonic components at $50 \mathrm{kHz}$ Two different bacteriophages have been reported, namely FR2 ( $\mathbf{Q})$ and $T 7(\triangle)$. 

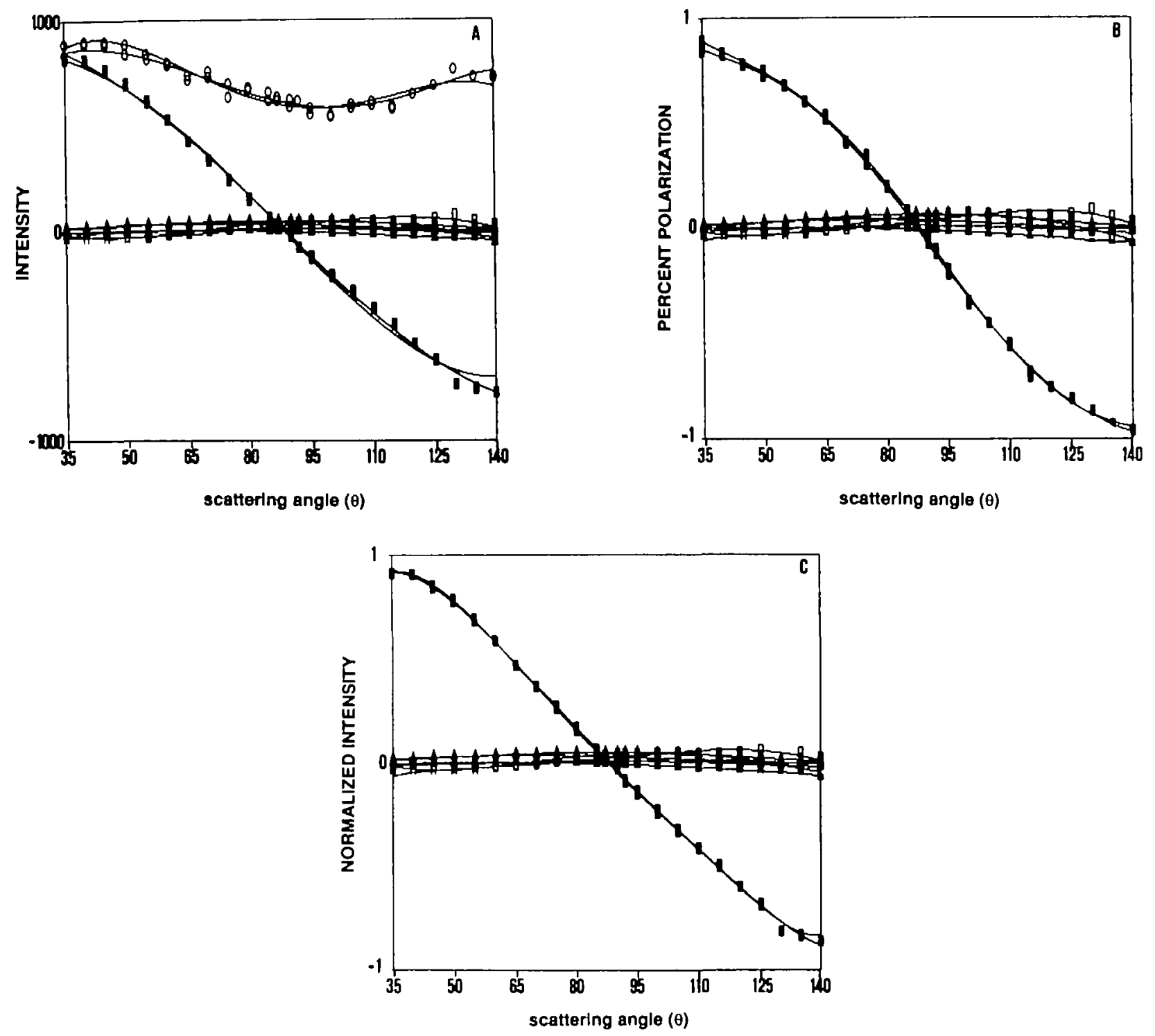

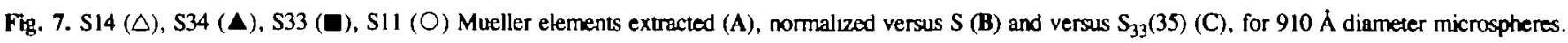

utilities, both data and graphic oriented; hardware utilities; pattern recognition modules for sample identification; operating system calls.

The source and executable MUCIDS files, with further hardware specifications, are available upon request at no cost, for scientific applications.

\section{Acknowledgements}

The authors would like to thank M.Bertolotto for his criticism in using the package, Dr M.Adami and Dr M.Sartore for their help in some software problems, and P.Milani for his tochnical support. Thanks are also due to Stefania Urbini for her graphic work. This work has been supported by Regione Liguria, Prodoati Gianni, National Research Council and Ministero Pubblica Istruzione.

\section{References}

Belmont,A., Zietz,S. and Nicolini,C. (1985) Differential scattering of circularly polarized light by chromatin modeled as a helical array of dielectric ellipsoids within the Born approximation. Biopolymers, 24, 1301-1321.

Bickel, W.S., Davidson,J.F., Huffmann, D.R. and Kilson, R. (1976) Application of polarization effects in light scattering: a new biophysical tool. Proc. Natl. Acad. Sci. USA, 73, 486-490.

Bickel,W.S. and Stafford,M.E. (1981) Polarized light scattering from biological systems: a technique for cell differentiation. J. Biol. Phys., 9, 53-66.

Bohren,C.F. and Huffmann,D.R. (1983) Absorption and Scattering of Light by Small Particles. Wiley, New York

Bustamante,C., Tinoco,I. and Maestre,M.R. (1983) Circular differential scattering can be an important part of the circular dichroism of macromolecules. Proc. Natl. Acad. Sci. USA, 80, 3568-3572.

Diaspro,A. and Nicolini,C.A. (1987a) Circular intensity differential scattering and chromatin-DNA structure. Cell Biophys., 10, 45-60.

Diaspro,A. and Nicolini,C. (1987b) CIDS: un metodo strumentale per la 
diagnostica di strutture microbiologiche, L'Elettrotecnica, 10, $1021-1026$.

Diaspro.A., Bertolotto,M., Vergani,L. and Nicolinı,C. (1990) Polarized light scattering of nucleosomes and polynucleosomes. In vitro and in situ studies. IEEE Trans. Biomed. Engng., in press.

Garab,G., Wells,S., Finzi,L. and Bustamante,C. (1988) Helically organized macroaggregates of pigment-protein complexes in chloroplasts: evidence from circular intensity differential scattering. Biochemistry, 27, 5839-5843.

Johnston,R.G., Shermila B. Singham and Salzman,G.C. (1988) Polarized light scattering. Comm. Mol. Cell. Biophys., 3, 171-192.

Maestre,M.F., Bustamante,C., Hayes,T.L., Subirana, J.A. and Tinoco,I. (1982) Differential scattering of circularly polarized light by helical sperm head from the octopus Eledone cirmosa. Nature, 298, 773-774.

Maestre,M.F., Salzman,G.C.. Tobey,R.A. and Bustamante,C. (1985) Circular dichroism studres on single Chinese hamster cell. Biochemistry, 24. $5152-5157$.

Nicolini,C. (1986) Biophysics and Cancer. Plenum, New York. pp. 267-268 and $382-404$.

Nicolini,C. and Kendall.F. (1977) Differential hight scattering in native chromatin: corrections and inferences combining meltung and dye-binding studies. A two order superhelical model. Physiol. Chem. Phys., 9, 265-283.

Nicolini,C., Baserga,R. and Kendall,F. (1976) DNA structure in sheared and unsheared chromatin. Science, 192, 796.

Nicolini,C., Diaspro,A. and Germano,P. (1987) The higher onder structure and dynamics of chromatin-DNA.In Nicolini,C. (ed.). Structure and Dynamics of Biopolymers. Martinus Nijhoff, Boston, MA, pp. 204-220.

Nicolini,C., Vergani,L.. Diaspro, A. and Scelza.P. (1988) Native chromatin and damage induced by nuclease. Biochem. Brophys. Res. Commun. 155,1396

Salzman,G.C. and Gregg.C.T. (1983) Current and experimental methods of rapid microbial identification. Biotechnology, 2, 243-248.

Zietz,S.. Belmont,A. and Nicolini,C. (1983) Differential scattenng of circularly polarized light as a unique probe of polynucleosome superstructures. Cell Biophys., 5, 163-187

Received on October 3, 1989; accepted on March 26, 1990

Circle No. 9 on Reader Enquiry Card 